\title{
GENERALIZATION OF LAGRANGE'S THEOREM
}

\author{
BY LOUIS WEISNER
}

1. Introduction. The following theorem due to Lagrange is of considerable importance in the theory of equations.

LAGRANGE'S THEOREM. If the group to which the rational function $\psi\left(x_{1}, \cdots, x_{n}\right)$ belongs is a subgroup of the group to which the rational function $\phi\left(x_{1}, \cdots, x_{n}\right)$ belongs, then $\phi$ equals a rational function of $\psi$ and the elementary symmetric functions of the variables $x_{1}, \cdots, x_{n}$.

In this paper I prove a similar theorem for sets of variables.

2. Notation and Definitions. Consider the $n$ sets of $m$ variables $x_{1 i}, x_{2 i}, \cdots, x_{m i}(i=1, \cdots, n)$, which may be regarded as coordinates of $n$ points in $m$-space. By a permutation of these sets of variables we mean a permutation of the points. Thus a permutation which changes $x_{1 i}$ to $x_{1 j}$, also changes $x_{2 i}, \cdots, x_{m i}$ to $x_{2 j}, \cdots, x_{m j}$ respectively. It is simpler to regard the permutation as affecting the second subscripts of the variables, with the above notation, than as affecting the $x$ 's.

A function $\phi\left(x_{11}, x_{21}, \cdots, x_{m 1} ; \cdots ; x_{1 n}, x_{2 n}, \cdots, x_{m n}\right)$ is said to belong to a substitution group $G$ on the symbols $1,2, \cdots, n$, if $\phi$ is unaltered by every substitution of $G$ and by no substitution on these symbols not contained in $G$. There exist functions which belong to a given substitution group. In fact, we can construct such functions involving only the variables $x_{11}, x_{12}, \cdots, x_{1 n}$.*

3. A Generalization. We proceed to prove the following generalization of Lagrange's Theorem.

* Netto, Substitutionentheorie und ihre Anwendung auf die Algebra, 1882 , p. 27. 
THEOREM. If the group to which the rational function $\psi\left(x_{11}, x_{21}, \cdots, x_{m 1} ; \cdots ; x_{1 n}, x_{2 n}, \cdots, x_{m n}\right)$ belongs, is a subgroup of the group to which the rational function $\phi\left(x_{11}\right.$, $\left.x_{21}, \cdots, x_{m 1} ; \cdots ; x_{1 n}, x_{2 n}, \cdots, x_{m n}\right)$ belongs, then $\phi$ equals a rational function of $\psi$ and the elementary symmetric functions of the sets of variables $x_{1 i}, x_{2 i}, \cdots, x_{m i},(i=1, \cdots, n)$.

It will suffice to consider the case $m=3$. The elementary symmetric functions of the $n$ triads of variables are defined by*

$$
\begin{array}{r}
p_{i j k}=\sum x_{11} x_{12} \cdots x_{1 i} x_{2,1+i} x_{2,2+i} \cdots x_{2, j+i} x_{3,1+i+j} x_{3,2+i+j} \cdots x_{3, k+i+s} \\
(i+j+k \leqq n) .
\end{array}
$$

With the aid of these functions, we can express any one of the variables $x_{1 i}, x_{2 i}, x_{3 i}$ as a rational function of any one of the others. In fact, $\uparrow$ we have

$$
\begin{aligned}
& x_{1 i}=\frac{p_{100} x_{3 i}{ }^{n-1}-p_{101} x_{3 i}{ }^{n-2}+p_{102} x_{3 i}{ }^{n-3}-\cdots}{n x_{3 i}{ }^{n-1}-(n-1) p_{001} x_{3 i}{ }^{n-2}+(n-2) p_{002} x_{3 i}^{n-2}-\cdots}, \\
& x_{2 i}=\frac{p_{010} x_{3 i}^{n-1}-p_{011} x_{3 i}{ }^{n-2}+p_{012} x_{3 i}{ }^{n-3}-\cdots}{n x_{3 i}{ }^{n-1}-(n-1) p_{001} x_{3 i}{ }^{n-2}+(n-2) p_{002} x_{3 i}{ }^{n-3}-\cdots} .
\end{aligned}
$$

Hence every function of the triads of variables can be expressed as a function of $x_{31}, x_{32}, \cdots, x_{3 n}$, with coefficients that belong to the symmetric group. In particular, suppose

$$
\begin{aligned}
& \psi\left(x_{11}, x_{21}, x_{31} ; \cdots ; x_{1 n}, x_{2 n}, x_{3 n}\right)=\psi_{1}\left(x_{31}, x_{32}, \cdots, x_{3 n}\right), \\
& \phi\left(x_{11}, x_{21}, x_{31} ; \cdots ; x_{1 n}, x_{2 n}, x_{3 n}\right)=\phi_{1}\left(x_{31}, x_{32}, \cdots, x_{3 n}\right) .
\end{aligned}
$$

Evidently $\psi$ and $\psi_{1}$ belong to the same group $H$, and $\phi$ and $\phi_{1}$ belong to the same group $G$. As $H$ is a subgroup of $G$ by hypothesis, it follows from Lagrange's Theorem, that $\phi$ equals a rational function of $\psi$ and the elementary symmetric functions $p_{001}, p_{002}, \cdots, p_{00 n}$. The theorem follows.

The University of Rochester

* See Bôcher, Higher Algebra, p. 252.

$\dagger$ Netto, Vorlesungen über Algebra, vol. II, p. 71. 